\title{
Multiplication and division of the orbital angular momentum of light with diffractive transformation optics
}

\author{
Gianluca Ruffato (1) ${ }^{1,2}$, Michele Massari ${ }^{2,3}$ and Filippo Romanato ${ }^{1,2,3}$
}

\begin{abstract}
We present a method to efficiently multiply or divide the orbital angular momentum (OAM) of light beams using a sequence of two optical elements. The key element is represented by an optical transformation mapping the azimuthal phase gradient of the input OAM beam onto a circular sector. By combining multiple circular-sector transformations into a single optical element, it is possible to multiply the value of the input OAM state by splitting and mapping the phase onto complementary circular sectors. Conversely, by combining multiple inverse transformations, the division of the initial OAM value is achievable by mapping distinct complementary circular sectors of the input beam into an equal number of circular phase gradients. Optical elements have been fabricated in the form of phase-only diffractive optics with high-resolution electron-beam lithography. Optical tests confirm the capability of the multiplier optics to perform integer multiplication of the input OAM, whereas the designed dividers are demonstrated to correctly split up the input beam into a complementary set of OAM beams. These elements can find applications for the multiplicative generation of higher-order OAM modes, optical information processing based on OAM beam transmission, and optical routing/switching in telecom.
\end{abstract}

\section{Introduction}

Since the seminal paper of Allen and coworkers ${ }^{1}$, light beams carrying orbital angular momentum (OAM) gave rise to a flourishing field of research, leading to a rich multiplicity of studies and applications: ${ }^{2}$ particle trapping, tweezing, and manipulation ${ }^{3}$, high-resolution microscopy $^{4,5}$, astronomical coronagraphy ${ }^{6}$, mode-division multiplexing ${ }^{7}$, and security ${ }^{8,9}$. Concurrently, several optical methods and devices have been described and engineered to tailor and control the OAM content of light beams, such as spiral phase plates ${ }^{10,11}$, computergenerated holograms ${ }^{12,13}, q$-plates ${ }^{14}$, transformation optics $^{15,16}$, and metasurfaces ${ }^{17}$. Basically, all those

Correspondence: Gianluca Ruffato (gianluca.ruffato@unipd.it)

'Department of Physics and Astronomy 'G. Galilei', University of Padova, via Marzolo 8, 35131 Padova, Italy

${ }^{2}$ LaNN, Laboratory for Nanofabrication of Nanodevices, EcamRicert, Corso StatiUniti 4, 35127 Padova, Italy

Full list of author information is available at the end of the article. methods rely on transferring an azimuthal phase gradient $\exp (i \ell 9)$ to the input beam, with $\vartheta$ being the polar angle on a plane transverse to the propagation direction and $\ell$ being the amount of OAM per photon in units of $\hbar$. In microscopy, a beam carrying OAM was used to suppress fluorescence around the dark central zone, providing subwavelength lateral confinement and consequent superresolution, as shown by the pioneering paper of Hell et al. $^{18}$. In the telecom field, the potentially unbounded state space provided by this even-unexploited degree of freedom offers a promising solution to increase the information capacity of optical networks ${ }^{19}$, both for free space $^{20}$ and optical fiber ${ }^{21}$ propagation. At present, it is urgent to further develop novel devices that can dynamically reconfigure and switch between distinct OAM modes $^{22,23}$ to fully exploit the extra degree of freedom provided by the OAM for both classical and quantum communications. The abovementioned conventional methods are useful for implementing only shift operations

\section{(c) The Author(s) 2019}

(c) (i) Open Access This article is licensed under a Creative Commons Attribution 4.0 International License, which permits use, sharing, adaptation, distribution and reproduction cc) in any medium or format, as long as you give appropriate credit to the original author(s) and the source, provide a link to the Creative Commons license, and indicate if changes were made. The images or other third party material in this article are included in the article's Creative Commons license, unless indicated otherwise in a credit line to the material. If material is not included in the article's Creative Commons license and your intended use is not permitted by statutory regulation or exceeds the permitted use, you will need to obtain permission directly from the copyright holder. To view a copy of this license, visit http://creativecommons.org/licenses/by/4.0/. 
on the OAM mode, i.e., addition or subtraction. On the other hand, it would be extremely beneficial to be able to either multiply or divide the OAM state for some applications, such as the multiplicative generation of highorder modes, optical switching, and routing ${ }^{24,25}$, and optical OAM-based information processing ${ }^{26,27}$.

To date, OAM multiplication and division have been implemented by means of bulky and sophisticated solutions ${ }^{28-30}$, which are barely suitable for integration and miniaturization owing to the presence of many optical elements. Multiplication has been performed ${ }^{28,29}$ by mapping the input azimuthal phase gradient into a linear phase gradient with a first $l o g$-pol sorter ${ }^{31,32}$, then creating multiple copies with an optical fan-out ${ }^{33}$, and finally wrapping the extended phase gradient into a doughnut shape with a second $l o g$-pol sorter, working in reverse, for a total of at least six optical elements, plus lenses in between for the Fourier transform and beam resizing. Division has been implemented in a similar manner ${ }^{29,30}$ by substituting the multiple-copy stage with a mask for the selection of only a fraction of the linear phase gradient in the input, at the expense, therefore, of a non-negligible amount of the input energy. In the quest for miniaturization, the implementation of the log-pol transformation stages in the form of diffractive optics ${ }^{34}$ and the integration of the copying/fractioning operation in the log-pol optics $^{35}$ could improve the compactness of the system. However, the number of optical operations, i.e., $\log$-pol mapping, phase-resizing, and inverse transformation, would remain the same.

Here, we present a completely different method, which basically preserves the axial symmetry and avoids the limitations of the log-pol coordinate-change approach. The key element is represented by an optical transformation that maps the azimuthal phase gradient of the input OAM beams into a circular sector. By combining multiple circular-sector transformation onto a single optical element, it is possible to multiply the input OAM by mapping its phase onto complementary circular sectors. Conversely, by combining multiple inverse transformations, it is possible to map different complementary sectors of the input beam into an equal number of circular phase gradients, thus achieving a division of the initial OAM. These operations can be realized by a sequence of only two elements, performing the optical transformation of the beam and the required phase correction. This approach allows for the multiplication and division of the OAM in a compact manner, remarkably reducing the number of optical operations and the total number of optical elements and therefore providing a final significant increase in the optical efficiency. The designed optical elements have been fabricated in the form of phase-only diffractive optics with high-resolution electron-beam lithography and optically characterized to demonstrate the expected capability to either multiply or divide the OAM of the input beam. In addition, a compact optical architecture has been presented to arrange the two optical elements required for optical transformation and phase correction onto the same substrate, further improving alignment and miniaturization.

\section{Results \\ Theory and simulation}

In the following paragraphs, the theory underlying multiplication and division with transformation optics is presented and described. At first, the key element of these operations, i.e., the circular-sector transformation, is introduced and developed in the paraxial approximation. Therefore, the possibility of combining many circularsector transformations to either multiply or divide the OAM content of an input beam is shown, and the phase patterns of the corresponding optical elements are calculated.

\section{Circular-sector transformation}

The optical layout of the system consists of a cascade of two optical elements: the former performs a conformal optical transformation, whereas the latter corrects the phase distortion owing to the different paths traveled by the distinct points of the beam and restores the desired phase profile. Conversely, owing to the invariance of the light path for time reversal, the second optical element performs the inverse optical transformation. The key element of OAM multiplication and division is represented by an optical transformation performing a conformal mapping of the whole circle onto a circular sector (Fig. 1a, d $)^{36}$. By indicating with $(r, 9)$ the polar coordinates on the input plane and with $(\rho, \phi)$ the polar reference frame on the second plane, this transformation operates a rescaling of the azimuthal coordinate:

$$
\phi=\frac{\vartheta}{n}
$$

After imposing the condition of conformity, the new radial coordinate is given by:

$$
\rho=a\left(\frac{r}{b}\right)^{-\frac{1}{n}}
$$

with $a$ and $b$ being scaling parameters. By applying the previous transformation, an azimuthal phase gradient is mapped conformally onto a circular sector with amplitude $2 \pi / n$. To calculate the phase pattern of an optical element performing this transformation in the paraxial regime, we apply the stationary phase approximation ${ }^{37}$ to the Fresnel integral. The field $U(\rho, \phi)$ after a propagation length $f$ along the coordinate $z$ for an input plane-wave 


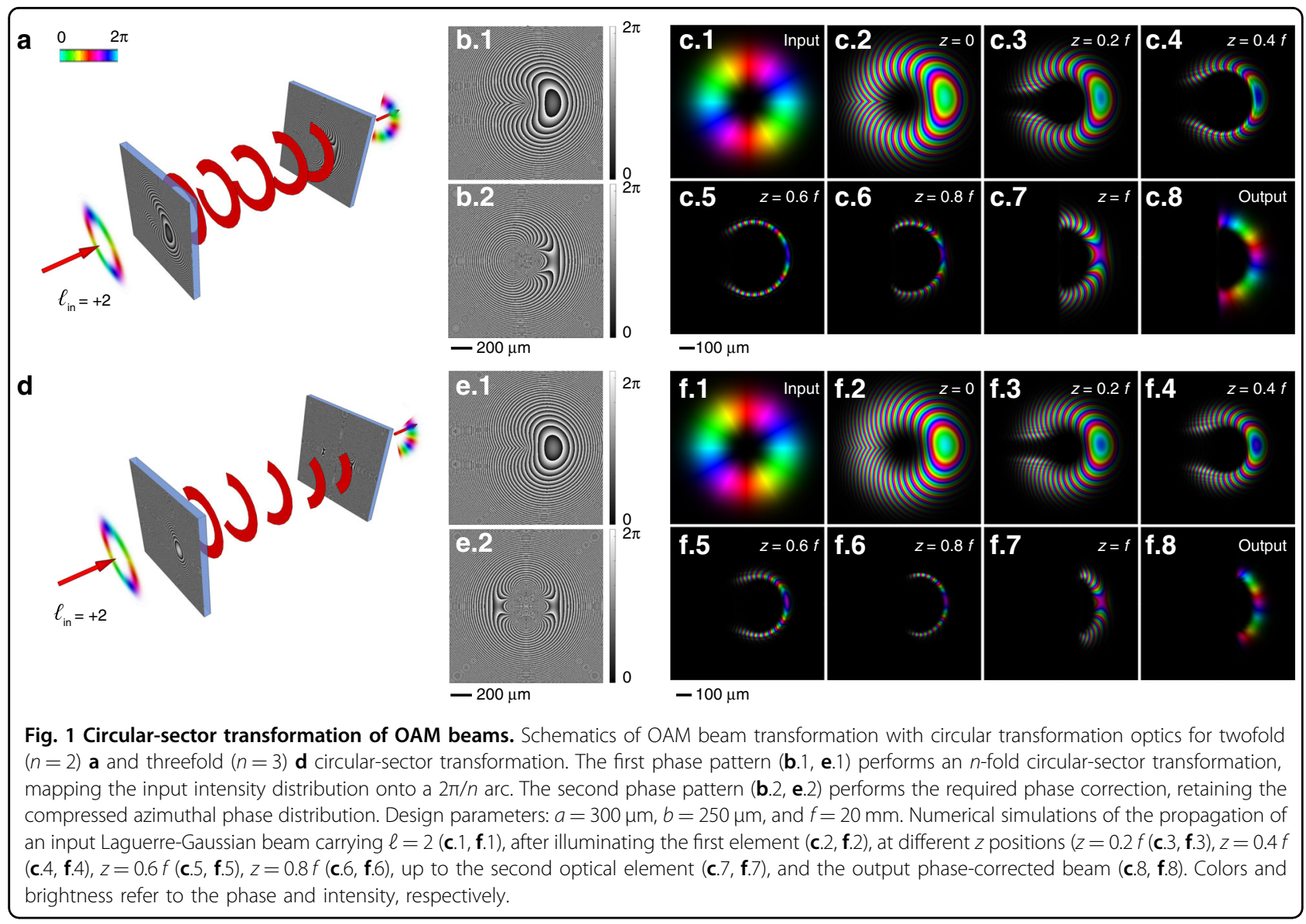

illuminating a phase-only optical element with phase function $\Omega_{S, n}$, located at $z=0$, is given by:

$$
U(\rho, \phi)=\frac{e^{i \kappa^{\frac{\rho^{2}}{2 f}}}}{i \lambda f} \iint e^{i \Omega_{S, n}(r, \vartheta)} e^{i k_{2 f}^{\frac{r^{2}}{2 f}}} e^{-i k_{f}^{\frac{p p}{f}} \cos (\vartheta-\phi)} r d r d \vartheta
$$

According to the stationary phase approximation ${ }^{37}$, the integral solution reduces to find the saddle points of the phase function in Eq. (3):

$$
\Phi(r, \vartheta)=\Omega_{S, n}(r, \vartheta)+k \frac{r^{2}}{2 f}-k \frac{r \rho}{f} \cos (\vartheta-\phi)
$$

The stationary condition $\nabla \Phi=0$ leads to a system of partial derivatives of $\Omega_{S, n}$ that is unknown. Substituting the transformation relations, i.e., Eqs. (1) and (2), into the partial derivatives of Eq. (4) and imposing the stationary condition, we obtain:

$$
\left\{\begin{array}{l}
\frac{\partial \Omega_{S, n}}{\partial r}+k \frac{r}{f}-k \frac{a}{f}\left(\frac{r}{b}\right)^{-\frac{1}{n}} \cos \left(\vartheta-\frac{\vartheta}{n}\right)=0 \\
\frac{\partial \Omega_{S, n}}{\partial \vartheta}+k \frac{a r}{f}\left(\frac{r}{b}\right)^{-\frac{1}{n}} \sin \left(\vartheta-\frac{9}{n}\right)=0
\end{array}\right.
$$

After integrating, we obtain the analytical expression for the phase function in the paraxial regime:

$$
\Omega_{S, n}(r, \vartheta)=\frac{2 \pi a b}{\lambda f}\left(\frac{r}{b}\right)^{1-\frac{1}{n}} \cdot \frac{\cos \left[\vartheta\left(1-\frac{1}{n}\right)\right]}{1-\frac{1}{n}}-k \frac{r^{2}}{2 f}
$$

The corresponding phase corrector can be calculated by applying the same method to the inverse transformation. We obtain:

$$
\Omega_{P C, n}(\rho, \phi)=\frac{2 \pi a b}{\lambda f}\left(\frac{\rho}{a}\right)^{1-n} \cdot \frac{\cos [\phi(1-n)]}{1-n}-k \frac{\rho^{2}}{2 f}
$$

which is basically the expression in Eq. (6) after the substitutions $b \leftrightarrow a, n \rightarrow 1 / n$, and $(r, \vartheta) \rightarrow(\rho, \phi)$. Further details regarding the calculations of Eq. (6) and Eq. (7) are provided in Supplementary Material S1.

In Fig. 1a, d, schematics of the OAM beam evolution in the case of circular-sector transformations by a factor of $n=2$ and $n=3$ are depicted, respectively. The phase gradient of the input OAM beam is mapped over half and onethird of the entire circle, respectively. Numerical simulations 


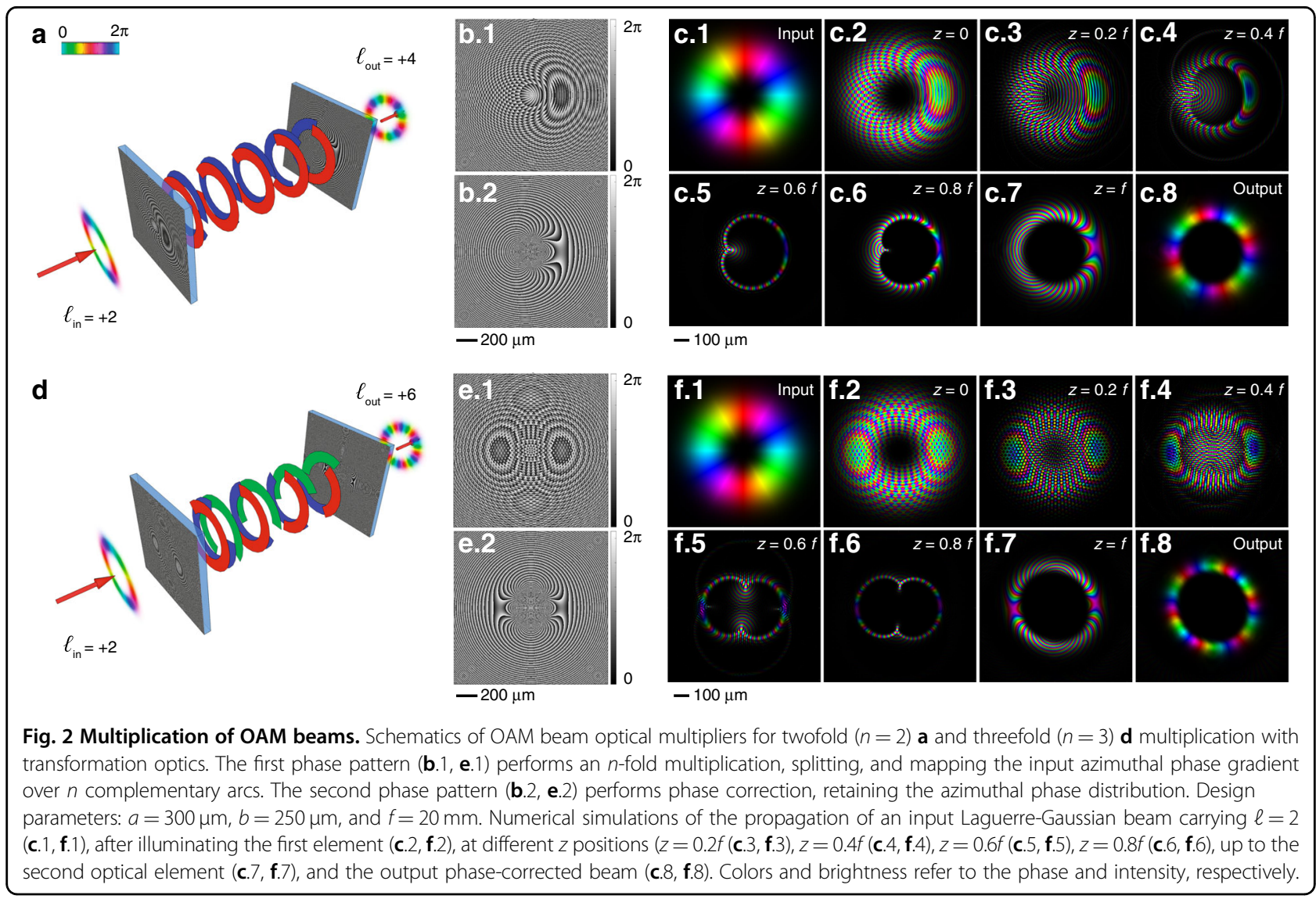

are also reported (Fig. 1c, f), showing the evolution of the beam after illuminating the first optical element and during propagation in the range of the focal length, where the second element is placed for phase correction.

\section{The OAM multiplier}

OAM multiplication by a factor $n$ basically consists of splitting the input beam into $n$ copies and mapping each one over a set of $n$ complementary circular sectors. Therefore, the phase pattern $\Omega_{M, n}$ of the multiplier is described by the superposition of $n$ circular-sector transformations $\left\{\Omega_{S, n}{ }^{(j)}\right\}, j=1, \ldots, n$, mapping the input beam over the corresponding circular sectors with amplitude $2 \pi / n$ and centered on $\{(j-1) 2 \pi / n\}$ :

$$
\Omega_{M, n}(r, \vartheta)=\arg \left\{\sum_{j=1}^{n} e^{i \Omega_{S, n}^{(j)}}\right\}
$$

where:

$\Omega_{S, n}^{(j)}(r, \vartheta)=\frac{2 \pi a b}{\lambda f}\left(\frac{r}{b}\right)^{1-\frac{1}{n}} \cdot \frac{\cos \left[\vartheta\left(1-\frac{1}{n}\right)+(j-1) \frac{2 \pi}{n}\right]}{1-\frac{1}{n}}-k \frac{r^{2}}{2 f}$
As demonstrated in Supplementary Material S2, the phase corrector of an $n$-fold circular transformation does not change if a shift of $j 2 \pi / n$, where $j$ is an integer value, is performed on the azimuthal coordinate after rescaling. Therefore, all the circular-sector transformations $\left\{\Omega_{S, n}{ }^{(j)}\right\}$ described by Eq. (9) share the same pattern for the phase corrector, which is given again by Eq. (7). In fact, the phase-corrector phase patterns in Fig. 2b.2 and Fig. 2e.2 of the twofold and threefold multipliers, respectively, are the same as those shown in Fig. 1b.2 and Fig. 1e.2 for the constituent twofold and threefold circular-sector transformations, respectively.

In Fig. $2 \mathrm{a}, \mathrm{d}$, schematics of the beam evolution in the case of multiplication by a factor of $n=2$ and $n=3$ are depicted, respectively. In Fig. 2c, f, the corresponding numerical simulations are reported, providing the evolution of the beam in the range of the focal length, where the second element is placed for the phase correction. As expected, the input beam is split into $n$ equal contributions that are mapped onto complementary arcs, thus performing an $n$-fold multiplication of the input OAM. A conversion efficiency close to the unit can be achieved, which gradually decreases with increasing input OAM value (Supplementary Material S4) owing to a slight distortion of the output intensity ring distribution. In Fig. 
2f.8, a slight deformation of the output beam can be observed, the shape of which is not perfectly axially symmetric. As discussed and demonstrated in detail in Supplementary Material S3, this distortion is caused by the twisted wavefront of the input OAM beam. In fact, the phase patterns in Eqs. (6) and (7) are calculated in the stationary phase approximation, assuming a uniform phase front in the input. On the other hand, OAM beams are endowed with a peculiar azimuthal phase, which affects the final intensity distribution of the transformed beam. However, as shown in the Supplementary Material, this effect can be mitigated and the conversion efficiency can be optimized by properly designing the transformation optics in terms of the focal length $f$ and design parameters $a$ and $b$.

\section{The OAM divider}

It could be interesting to divide the input beam into a bunch of $n$ beams with OAMs equal to $1 / n$ the OAM of the input beam, as depicted in Fig. 3a, d, for the case of twofold $(n=2)$ and threefold $(n=3)$ division, respectively. The OAM division operation can be understood considering OAM multiplication, e.g., Fig. 2d, in reverse. In Fig. 2d, the output threefold OAM beam is the combination of three complementary circular sectors of size $2 \pi / 3$ that arise from the same number of combined threefold circular-sector transformations of the input beam (threefold multiplier). Thus, the input OAM beam carrying $\ell=2$ is split into three copies that are unwrapped and projected onto three complementary arcs to achieve the final $\ell=6$ OAM beam. Considering the optical path in reverse, the three complementary circular sectors with size $2 \pi / 3$ forming the OAM beam with $\ell=6$ are wrapped by a circular-sector transformation with $n=$ $1 / 3$ onto three OAM beams carrying the OAM value $\ell / 3=2$. By applying different tilt angles to the three arcs, it is possible to obtain three non-overlapping OAM beams as the output, as shown in Fig. 3d. Generalizing this approach to a division into $n$ OAM beams, this is possible by defining the phase pattern of the OAM divider in the following way:

$$
\Omega_{D, n}(r, \vartheta)=\arg \left\{\sum_{j=1}^{n} e^{i \Omega_{S, 1 / n, j}(r, \vartheta)} e^{i r \cdot \beta^{(j)} \cos \left(9-\vartheta^{(j)}\right)} \Phi_{n}^{(j)}(\vartheta)\right\}
$$

where $\Omega_{S, 1 / n, j}$ is the phase pattern of a $1 / n$-fold circularsector transformation centered on $(j-1) 2 \pi / n, j=1, \ldots, n$ :

$$
\Omega_{S, 1 / n, j}(r, 9)=\frac{2 \pi a b}{\lambda f}\left(\frac{r}{b}\right)^{1-n} \cdot \frac{\cos \left[\left(9-(j-1) \frac{2 \pi}{n}\right)(1-n)\right]}{1-n}-k \frac{r^{2}}{2 f}
$$

and $\Phi_{n}{ }^{(j)}$ is a phase mask selecting the desired circular sector with its center at $(j-1) 2 \pi / n$ and a size of $2 \pi / n$ :

$$
\Phi_{n}^{(j)}(\vartheta)=\Theta\left(\vartheta-\frac{2 \pi}{n}(j-1)+\frac{\pi}{n}\right) \Theta\left(\frac{2 \pi}{n}(j-1)+\frac{\pi}{n}-\vartheta\right)
$$

where $\Theta(\cdot)$ is the Heaviside function $(\Theta(x)=1$ when $x \geq 0$ and $\Theta(x)=0$ otherwise). According to Eq. (10), the total phase pattern of the OAM divider is basically the composition of $n$ complementary and non-overlapping phase patterns, each defined over a circular sector spanning an angle equal to $2 \pi / n$ and centered on $(j-1)$ $2 \pi / n, j=1, \ldots, n$. Specifically, each zone is illuminated by $1 / n$ of the input beam, and it is designed to wrap the impinging arc around the entire circle ( $1 / n$-fold circularsector transformation). In addition, spatial frequency carriers $\left\{\left(\beta^{(j)}, \vartheta^{(j)}\right)\right\}$ are introduced in Eq. (10), to spatially separate the $n$ output beams and locate them at defined positions $\left(\rho^{(j)}, \phi^{(j)}\right)$ in the focal plane according to:

$$
\left\{\begin{array}{l}
\rho^{(j)}={ }_{k}^{f} \beta^{(j)} \\
\phi^{(j)}=9^{(j)}=(j-1) \frac{2 \pi}{n}
\end{array}\right.
$$

where $j=1, \ldots, n$ and $f$ is the focal length of the optical transformation. Then, the phase corrector for the divider in Eq. (10) is given by the composition of $n$ complementary and non-overlapping phase correctors, each defined over a circular sector spanning an angle equal to $2 \pi / n$ and centered at the positions given by $\left(\rho^{(j)}, \phi^{(j)}\right)$ :

$$
\begin{gathered}
\Omega_{D-P C, n}(\rho, \phi)= \\
\arg \left\{\sum_{j=1}^{n} e^{i \Omega_{P C, 1 / n, j}\left(\rho^{\prime}, \phi^{\prime}\right)} \Phi_{n}^{(j)}(\phi)\right\}
\end{gathered}
$$

where $\left(\rho_{\mathrm{j}}^{\prime}, \phi_{\mathrm{j}}^{\prime}\right)$ are polar coordinates centered on $\left(\rho^{(j)}, \phi^{(j)}\right)$ given by Eq. (13), and:

$$
\begin{gathered}
\Omega_{P C, 1 / n, j}\left(\rho_{j}^{\prime}, \phi_{j}^{\prime}\right) \\
=\frac{2 \pi a b}{\lambda f}\left(\frac{\rho_{j}^{\prime}}{a}\right)^{1-\frac{1}{n}} \cdot \frac{\cos \left[\left(\phi_{j}^{\prime}-(j-1) \frac{2 \pi}{n}\right)\left(1-\frac{1}{n}\right)\right]}{1-\frac{1}{n}}-k \frac{\rho^{2}}{2 f}
\end{gathered}
$$

In Fig. 3c, f, numerical calculations are reported in the case of $n=2$ and $n=3$, showing the evolution of the beam in the range of the focal length. As expected, the input beam is split into $n$ equal contributions that are mapped onto distinct circles, thus performing an $n$-fold division of the input OAM into a set of $n$ OAM beams. In principle, the OAM divider can be generalized and properly designed to decompose the input OAM into 


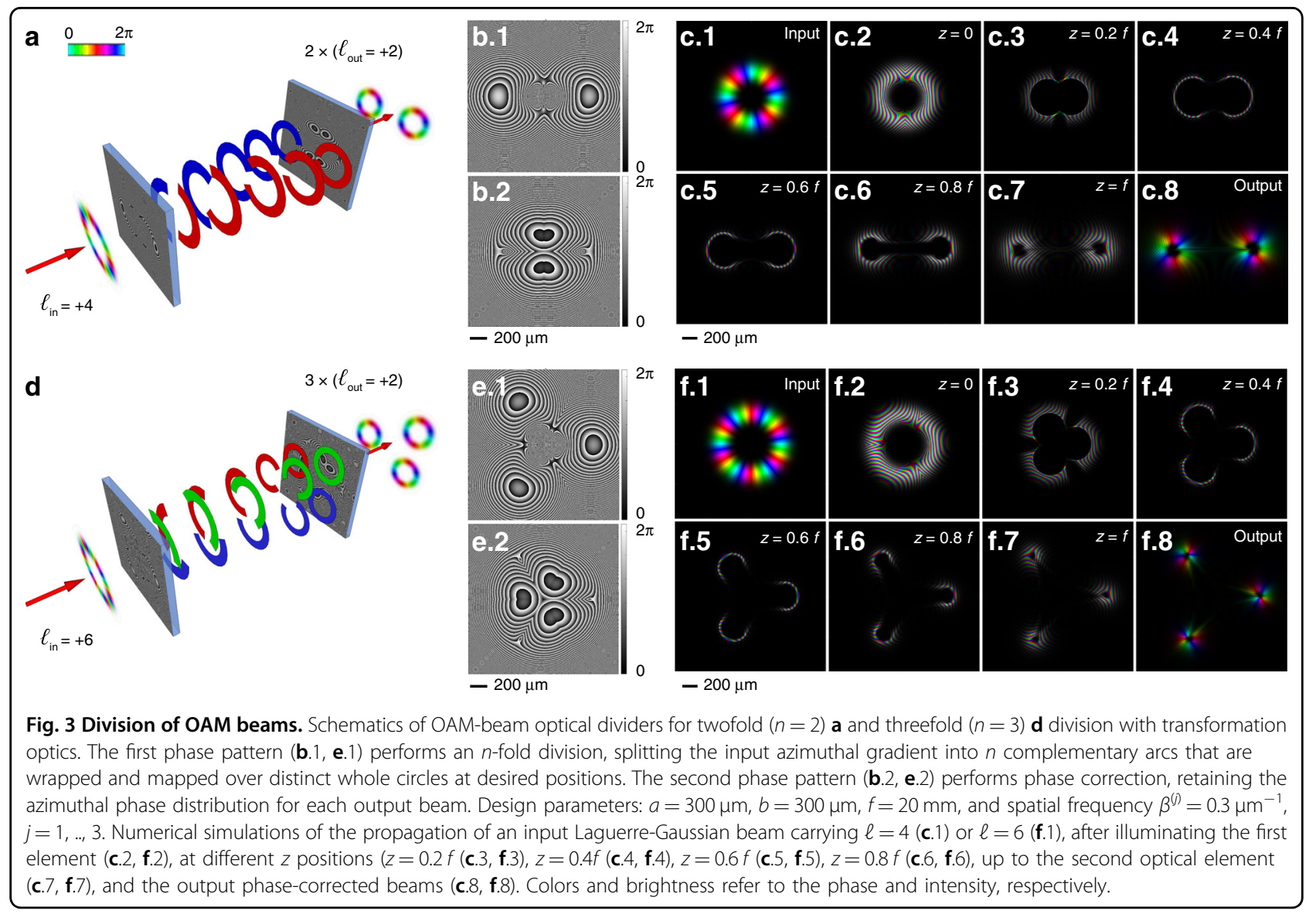

many beams carrying different values of the OAM and whose total sum equals the OAM value of the input beam.

\section{Optical element design}

A limit of performing optical operations with transformation optics is the need for at least two optical elements, i.e., transformer and phase corrector. As widely experienced in the case of OAM sorting with a log-pol transformation, the presence of two confocal elements to be perfectly aligned, coaxial and coplanar, could require arduous efforts for alignment and could be detrimental to miniaturization and integration. To simplify the alignment process and improve the compactness of the optical architecture, we designed the optical configuration to incorporate the two elements onto a single optical platform. In this configuration, the substrate is illuminated twice: after crossing the first zone, while performing multiplication/division, the beam is back-reflected with a mirror, placed at half the focal length, and made to impinge on the second region providing the phase correction. By adding a tilt term to the first phase pattern, the back-reflected beam does not overlap with the input one, and the two optical elements, i.e., transformer and phase corrector, can be fabricated side-by-side on the same substrate. This solution makes the alignment operation significantly easier, as the number of degrees of freedom is remarkably reduced and the two elements are by-design aligned and parallel to each other. We fabricated compact diffractive optics performing either twofold or threefold multiplication and division. The focal length was fixed to $20 \mathrm{~mm}$; therefore, the distance between the optics and the mirror was reduced to $1 \mathrm{~cm}$. Adding to the first pattern a spatial frequency $\beta$ of $\sim 0.5 \mu \mathrm{m}^{-1}$, the centers of the two phase elements were found to be $\sim 1 \mathrm{~mm}$ from each other. For the multiplier, this result is achieved by adding a phase term $\beta x$ to the phase in Eq. (8) and centering the phase corrector at a distance $\beta f / k$ from the multiplier center. As far as the divider is concerned, as shown in Eq. (10), spatial frequency carriers are already included to separate the different OAM beams in the output; therefore, it is sufficient to choose the moduli $\beta^{(j)}$ to avoid overlap of the phase-correcting zones with the divider pattern. The radius of the first zone was set at approximately $600 \mu \mathrm{m}$, whereas the radii of the phase correctors were chosen to avoid overlapping the first pattern (see Fig. 4 and Fig. 5). In more detail, for both multipliers, we set the parameters $a$ and $b$ to $300 \mu \mathrm{m}$ and $250 \mu \mathrm{m}$, respectively, whereas for the dividers we chose the values $a=250 \mu \mathrm{m}$ and $b=400 \mu \mathrm{m}$. 

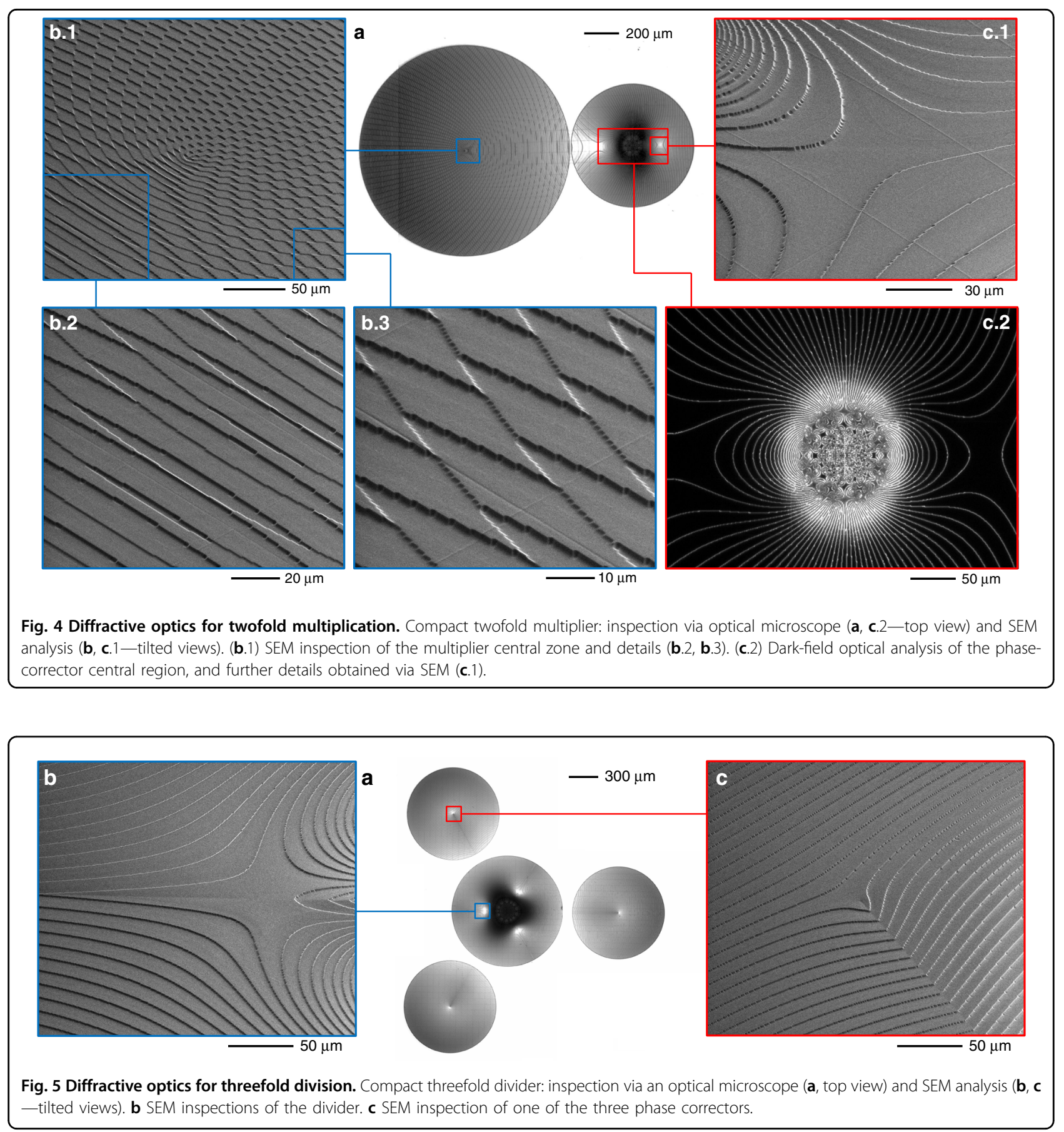

\section{Electron-beam lithography}

The designed optical elements were fabricated as surface-relief phase-only diffractive optics using highresolution electron-beam lithography (EBL) over a resist layer. By locally controlling the released electronic dose, a different dissolution rate is induced point-by-point on the exposed polymer with a resolution of few nanometers, giving rise to a spatially variant resist thickness after the development process ${ }^{38}$. A dose-depth correlation curve (contrast curve) is required to calibrate the correct electron dose to assign to obtain the desired thickness. For a phase pattern $\Omega(x, y)$, the depth $d(x, y)$ of the exposed zone for normal incidence in air is given by:

$$
d(x, y)=\frac{\lambda}{n_{R}(\lambda)-1} \cdot \frac{2 \pi-\Omega(x, y)}{2 \pi}
$$

where $\lambda$ is the incident wavelength and $n_{R}(\lambda)$ is the corresponding refractive index of the resist. In this work, 


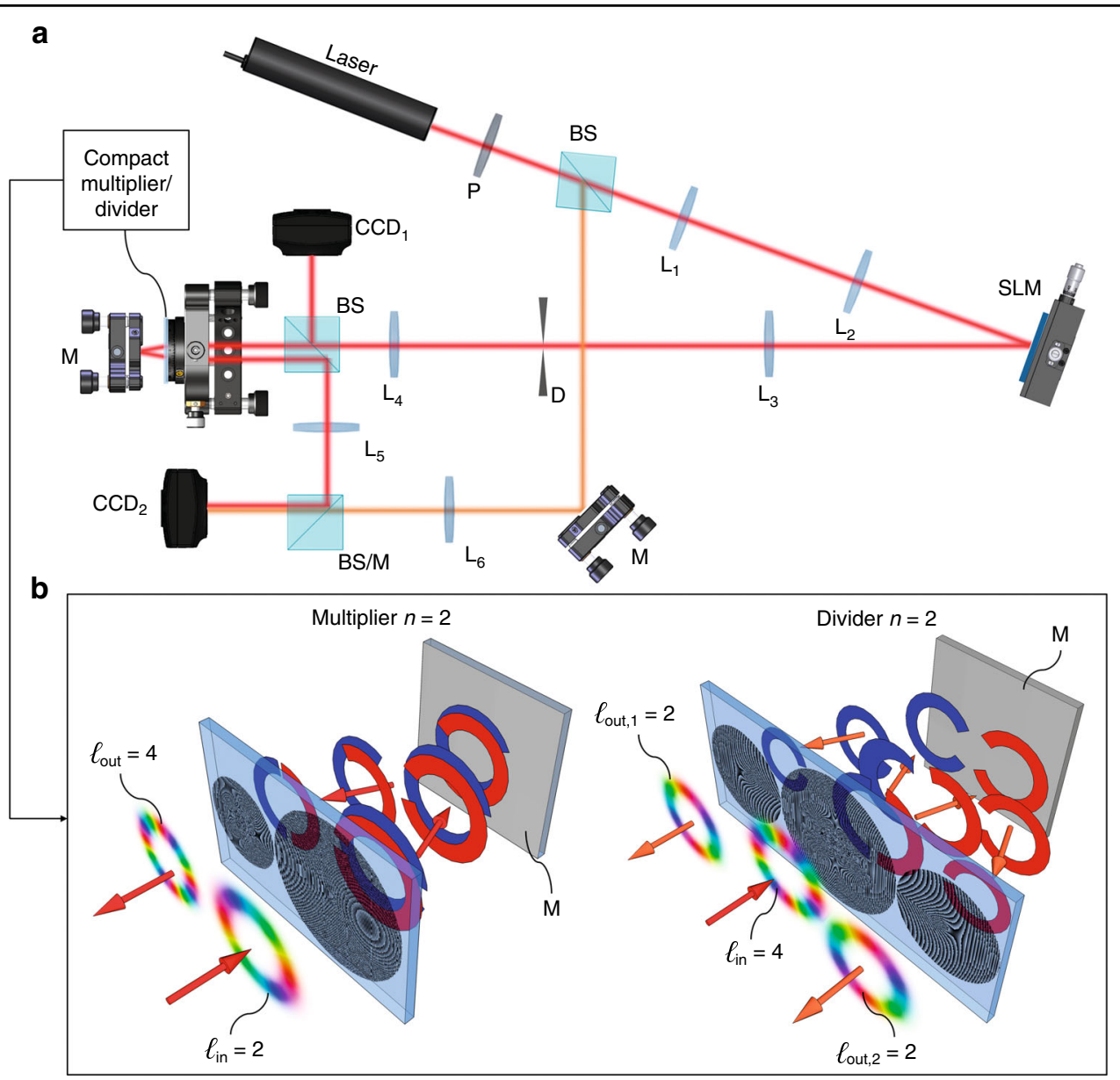

Fig. 6 Scheme of the experimental setup. a Scheme of the experimental setup used for the optical characterization of the fabricated dividers and multipliers. The laser beam is linearly polarized $(P)$ and expanded $\left(f_{1}=3.5 \mathrm{~cm}, f_{2}=12.5 \mathrm{~cm}\right.$ ). A beam splitter (BS) is used to generate a reference Gaussian beam for the interferometric bench. The SLM first order is filtered (D) and resized $\left(f_{3}=25.0 \mathrm{~cm}, f_{4}=12.5 \mathrm{~cm}\right)$ before illuminating the optical element as in $\mathbf{b}$. A mirror (M) is used for back-reflection onto the phase-correcting pattern. A second beam splitter is used both to check the input beam and collect the output on two different cameras. The second camera is placed on the back focal plane of a fifth Fourier lens $\left(f_{5}=10.0 \mathrm{~cm}\right)$, and it is used to collect the output beam intensity and its interferogram $\left(f_{6}=20.0 \mathrm{~cm}\right)$. b Compact optical configurations for the twofold multiplier and the twofold divider. The two elements, i.e., tilting divider/multiplier and phase corrector, are placed on the same substrate, and a mirror (M) is used for back-reflection.

all diffractive optics were fabricated by patterning a layer of negative resist (AR-N 7720.30, Allresist) spin-coated onto a $1.1 \mathrm{~mm}$-thick ITO-coated soda lime float glass substrate. At the experimental wavelength of the laser $(\lambda=632.8 \mathrm{~nm})$, the refractive index of the resist polymer was assessed to be $n_{\mathrm{R}}=1.679$. From Eq. (16), the maximal depth of the surface-relief pattern was found to be $928.5 \mathrm{~nm}$, with a thickness resolution of $\Delta d=3.64 \mathrm{~nm}$. The quality of the fabricated phase-only optical elements was assessed with scanning electron microscopy (SEM) and optical microscopy. In Fig. 4, SEM inspections are reported, referring to the compact two fold multiplier. In Fig. 5, the inspections refer to the compact threefold divider.

\section{Optical characterization}

The optical response of the fabricated optical elements has been analyzed for illumination under integer OAM beams generated with an LCoS spatial light modulator (SLM) using a phase and amplitude modulation technique $^{39}$. Once properly filtered and resized, the OAM beam illuminated the first zone of the sample, performing either $n$-fold multiplication or division, as shown in the experimental layout of the optical bench in Fig. 6a. A mirror was placed on a kinematic mount, and its position could be finely controlled with a micrometric translator to set the distance from the multiplier/divider to equal to half the focal length of the first element, i.e., $1 \mathrm{~cm}$. After back reflection, the transformed beam illuminated the optics again on the phase-correcting zones, as shown in the schemes in Fig. 6b, and the far-field was collected by a second camera placed at the back focal plane of a Fourier lens. To check the OAM content of the output beams, a Mach-Zehnder interferometric bench was added to the described optical setup, as depicted in Fig. 6 . 


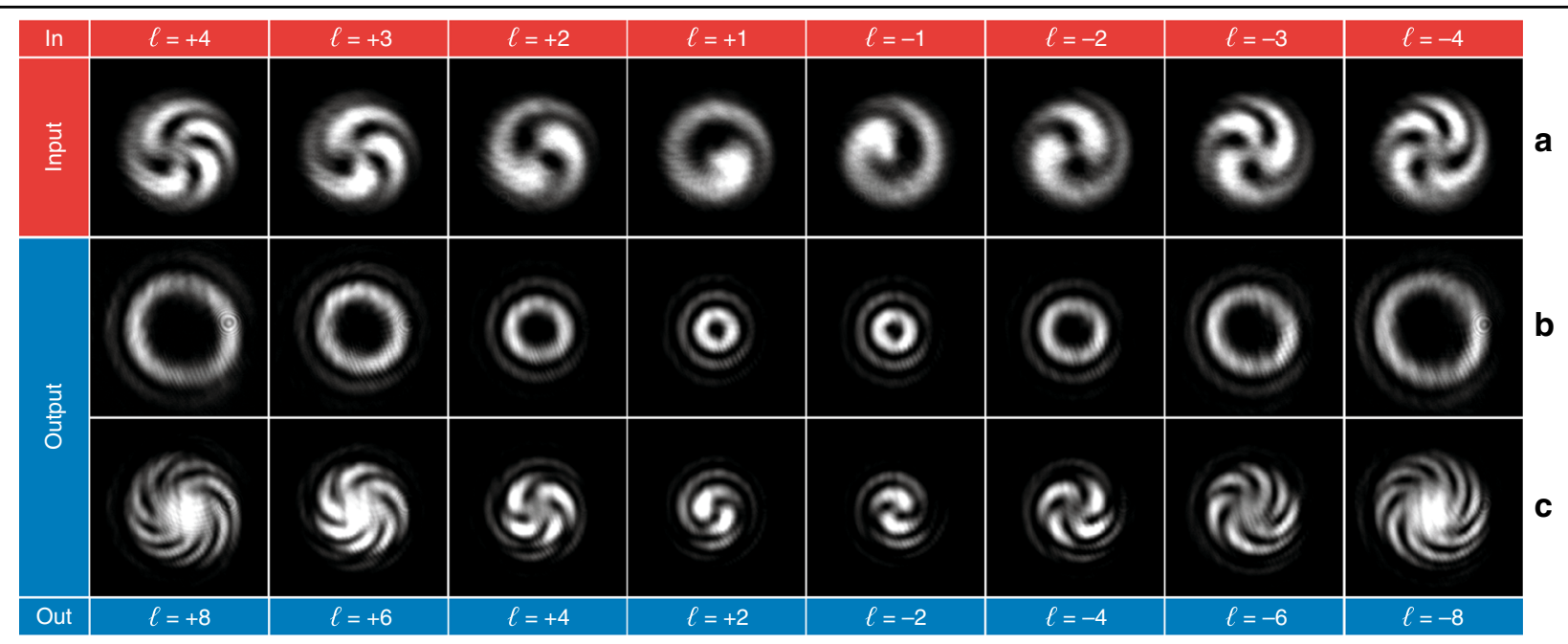

Fig. 7 Optical characterization of the twofold multiplier for input OAM beams with $\ell$ values in the range from -4 to $+4,0$ excluded. $\mathbf{a}$ Interferogram of the input OAM beams. Intensity pattern $\mathbf{b}$ and interferogram $\mathbf{c}$ of the output beam. As expected, the number of spiral arms in the output interference pattern is equal to twice the number in the input interferogram.

The optical response of the fabricated optics was tested for the multiplication and division of optical beams with integer OAM values. The OAM of the output beam was analyzed from the interference pattern, with a reference arm obtained by splitting the Gaussian output of the laser. By counting the number of arms in the generated spiralgrams, it is possible to infer the OAM of the output beam and prove the expected multiplication or division of the initial OAM state. The optical characterization of the fabricated samples confirmed the expected capability to perform optical multiplication and division of the OAM of the input beam.

In Fig. 7, the optical characterization is reported for the twofold multiplier. The optical response was characterized for input OAM beams with $\ell$ in the range from -4 to +4 (Fig. 7a), 0 excluded. As expected, the interference pattern of the output beam shows twice the number of spiral arms of the input interference pattern, confirming the duplication of the input OAM value (Fig. 7c). The same analysis was performed for the threefold multiplier for input OAM values in the range from -3 to $+3,0$ excluded (Fig. 8a). As shown in Fig. 8c, the number of output arms in the interferograms is three times the number of input arms, as expected.

In Fig. 9, the experimental output of the twofold divider is reported for input OAM beams with even $\ell$ values in the range from -8 to $+8,0$ excluded (Fig. 9a). As expected, the input beam is split into two output beams (Fig. 9b), with an OAM equal to half the input value. In Fig. 9c, the interferogram is reported for only one out of the two beams, and the interference pattern confirms the capability of the designed optics to divide the input OAM into two. The same analysis was performed for the threefold divider, as reported in Fig. 10. In this case, the output is composed of three OAM beams (Fig. 10b), each carrying one-third of the input OAM, as demonstrated by the experimental interferograms (Fig. 10c) for input $\ell$ in the range from -9 to +9 , with a step of 3,0 excluded (Fig. 10a).

\section{Discussion}

In this paper, we presented for the first time a comprehensive work of simulation, fabrication, and optical characterization of a new type of optical device performing the division and multiplication of the orbital angular momentum of light. With respect to previous demonstrations, these optical operations were performed here in a compact and efficient configuration by means of a cascade of only two confocal optical elements. The former performs the desired optical transformation, i.e., $n$-fold multiplication or division of the input beam, whereas the latter introduces the required phase correction to correct the phase distortion accumulated during beam propagation. Both multiplication and division were implemented with a superposition of many circular-sector transformations. Specifically, multiplication by a factor $n$ was realized by splitting the input OAM beam into $n$ copies and mapping the whole azimuthal gradients into $n$ complementary circular sectors, thus obtaining a final azimuthal gradient that is $n$ times the input one. Conversely, OAM division was achieved by splitting the input azimuthal gradient into $n$ complementary circular sectors and mapping them onto $n$ complete circles at distinct positions.

The phase patterns of the phase-only optical elements performing twofold and threefold division/multiplication 


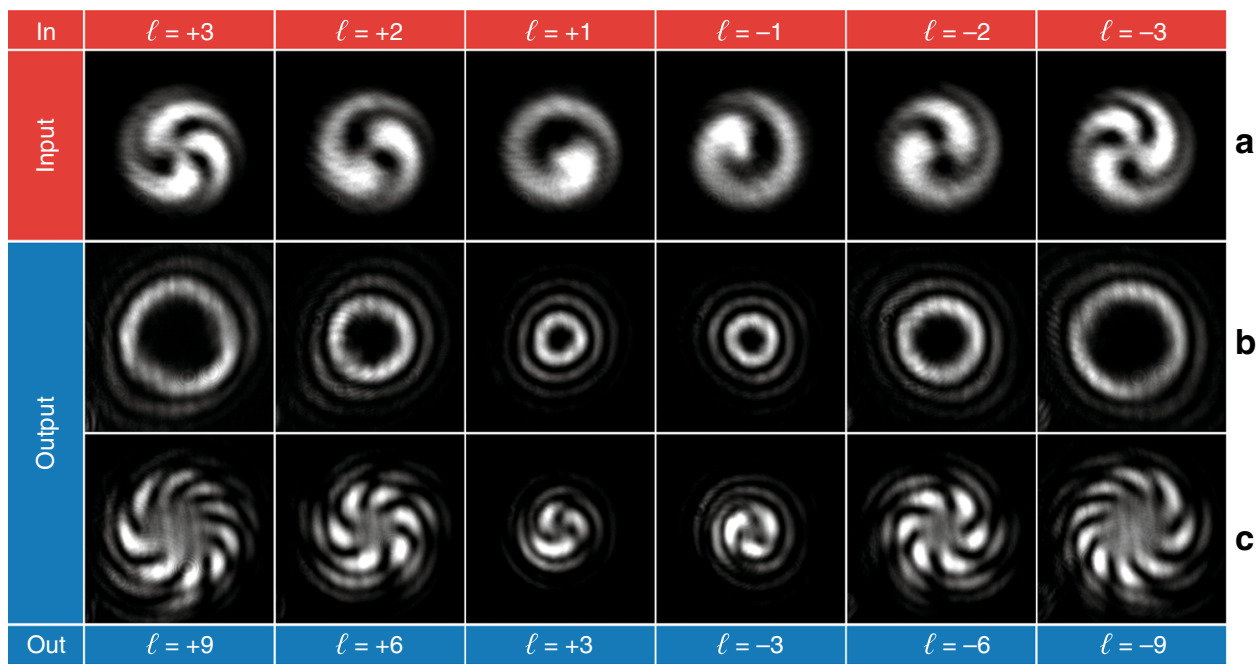

Fig. 8 Optical characterization of the threefold multiplier for input OAM beams with $\ell$ values in the range from -3 to $+3,0$ excluded. $\mathbf{a}$ Interferogram of the input OAM beams. Intensity pattern $\mathbf{b}$ and interferogram $\mathbf{c}$ of the output beam. As expected, the number of spiral arms in the output interference pattern is equal to three times the number in the input interferogram.

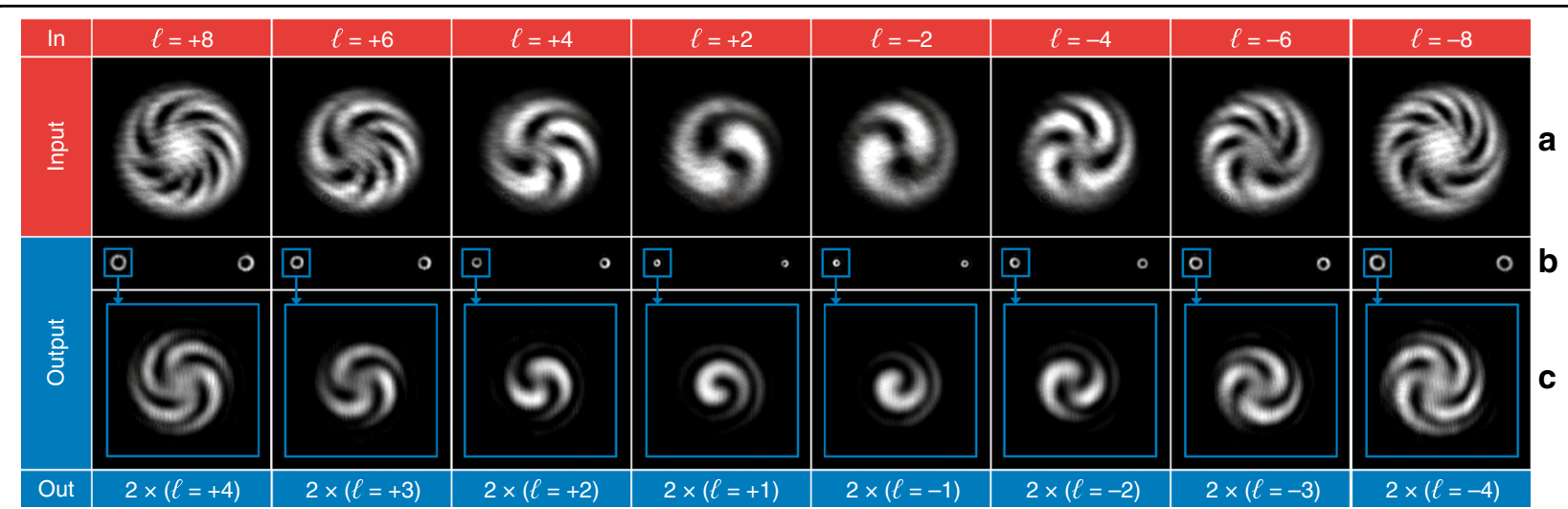

Fig. 9 Optical characterization of the twofold divider for input OAM beams with even $\ell$ values in the range from -8 to $+8,0$ excluded. a Interferogram of the input OAM beams. Intensity pattern $\mathbf{b}$ and interferogram $\mathbf{c}$ of the output beam. As expected, the number of spiral arms in the output interference pattern is equal to half the number in the input interferogram.

were calculated in the paraxial approximation and implemented in the form of diffractive optics fabricated with high-resolution electron-beam lithography. Furthermore, the compactness of the optical system was further improved by fabricating the two optical elements, i.e., multiplier/divider and the corresponding phase corrector, side-by-side on the same substrate, therefore reducing the number of degrees of freedom and significantly improving the alignment and miniaturization. This outcome is achievable by introducing a tilt in the first optical element and using a mirror for back-reflection onto the second pattern performing phase correction. The optical characterization with input integer OAM values confirmed the expected capability of the fabricated samples to perform either multiplication or division of the OAM content of the input beams.
As discussed in ref. ${ }^{40}$ in the case of the log-pol optical transformer, the phase elements are designed to work perfectly for collimated beams in the input, i.e., plane wavefronts, in which all light rays illuminate the first element with normal incidence. Otherwise, it is required that the angular deviation introduced by the transformer, which is given by $|\nabla \Omega| / k$, dominates over any deviation from normal incidence with which the rays arrive. Irrespective of their divergence, beams carrying OAM are never plane waves, as it is the azimuthal component of the Poynting vector that originates the orbital angular momentum in the propagation direction, introducing a local skew angle around $\ell / k r^{41}$. It follows that for the multiplier/divider optics to work efficiently for an OAM beam of radius $r$, it is required that $\ell / k r<<a(r / b)^{-1 / n} / f$, which provides the condition $\ell<<k a b(r / b)^{1-1 / n} / f$ (see 


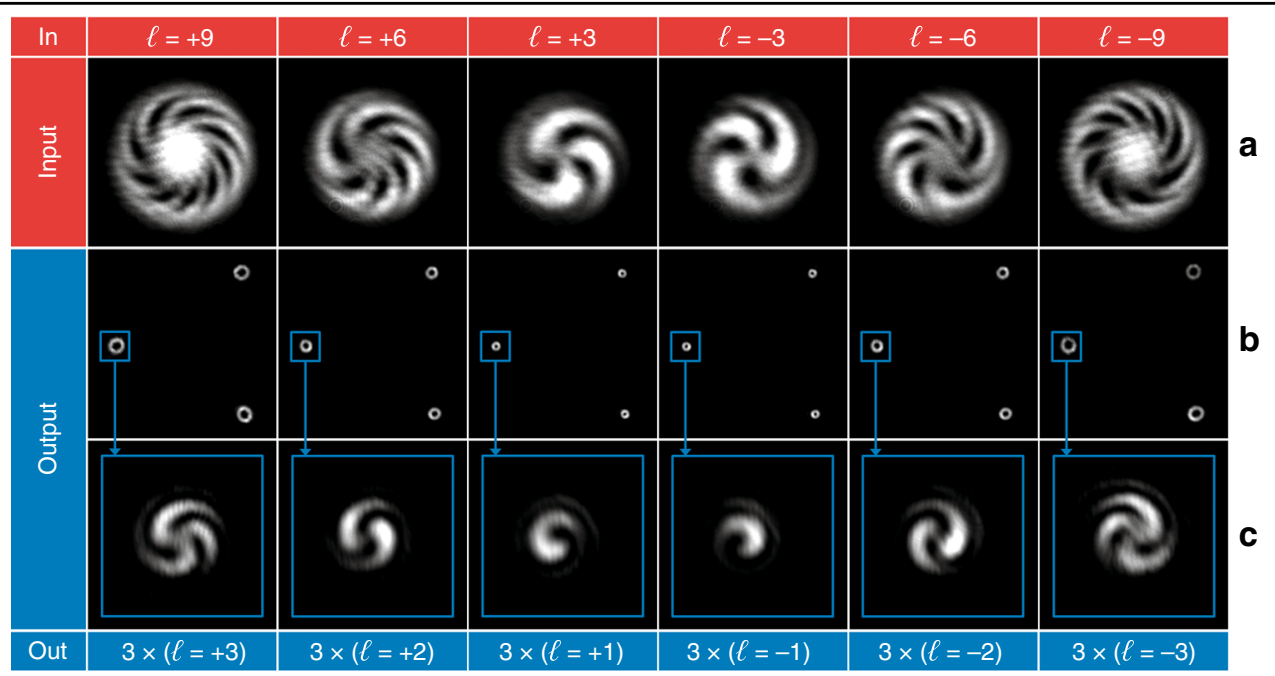

Fig. 10 Optical characterization of the threefold divider for input OAM beams with $\ell$ values in the range from -9 to +9, with a step of 3,0 excluded. a Interferogram of the input OAM beams. Intensity pattern $\mathbf{b}$ and interferogram $\mathbf{c}$ of the output beam. As expected, the number of spiral arms in the output interference pattern is equal to one-third the number in the input interferogram.

Supplementary Material S3 for more details on calculations). Therefore, by properly designing the optical elements in terms of the transformation parameters $(a, b)$ and focal length $f$, it is possible to increase the input OAM value, which can be either multiplied or divided with minimal distortion and maximal conversion efficiency.

So far, the possibility of increasing and decreasing the OAM content of a beam in a multiplicative manner was still missing; therefore, the framework offered by these optical elements can offer interesting and promising applications in a wide range of fields, both in the classical and single-photon regimes. OAM multiplication could find applications in the generation of high-order OAM states, for structured-light studies, or for incremental particle tweezing and trapping/manipulation. By using many low-order multipliers in cascades, it could be possible to easily increase the OAM by one order of magnitude or more. In this case, the design of the optics should be properly optimized to extend the OAM bandwidth and allow operation on high OAM values without dramatic distortions. By increasing the input OAM using a twofold or threefold multiplier before using a standard log-pol sorter, it could be possible to significantly enhance the cross-talk by improving the channel separation without the need for a fan-out optical operation. As a matter of fact, OAM multiplication and division are also expected to work for OAM-beam superposition (see Supplementary Material S4). Multipliers and dividers could represent the key elements needed to implement OAM algebra in optical boards performing computations with the OAM of light. Moreover, they can allow the compact and efficient implementation of the operations required in nextgeneration optical platforms performing switching and routing between OAM channels in mode-division multiplexing architectures. In this paper, OAM division was limited to an equalized set of output modes; however, it could be generalized for the generation of any complementary set of either integer or fractional OAM states.

The theory underlying the division and multiplication of azimuthal phase gradients, peculiar of modes endowed with orbital angular momentum, can be extended beyond the specific optical range of the presented study. For instance, the exploitation of mode-division multiplexing for high-capacity transmissions in free space has acquired increasing attention in the microwave regime, and the implementation of diffractive optics for OAM control and manipulation has recently been shown ${ }^{42}$. Moreover, the interest in physical states carrying OAM has recently encompassed other branches of physics by applying the results of optics to quantum mechanical wave functions describing massive particles (matter waves) ${ }^{43}$. In particular, the possibility to control and analyze the OAM of free-electron beams has recently been investigated with diffractive $^{44,45}$ or electrostatic phase ${ }^{46}$ elements. Therefore, we envisage that similar devices performing multiplication/division could be developed for studies concerning the multiplicative transformation of the OAM carried by twisted matter waves.

\section{Materials and methods}

\section{Numerical simulations}

A custom MATLAB code was implemented based on the convolution algorithm applied to the Fresnel diffraction integral ${ }^{47}$ to compute the propagation of an OAM beam impinging on the multiplier/divider phase pattern and its final phase and intensity distributions after 
illuminating the phase-corrector element. The phase patterns were defined over a mesh of $5121 \times 5121$ pixels with a resolution of $0.3125 \mu \mathrm{m}$. Simulations have been performed for several $n$-fold dividers and multipliers with different design parameters $(a, b)$ and focal length values $f$.

\section{Electron-beam lithography}

Diffractive optics were fabricated by patterning a layer of negative resist (AR-N 7720.30, Allresist), spin-coated on a $1.1 \mathrm{~mm}$-thick ITO-coated soda lime float glass substrate (resist thickness of $\sim 3 \mu \mathrm{m}$ ), and pre-baked for $30 \mathrm{~min}$ at $85^{\circ} \mathrm{C}$. The fabricated phase patterns were computed as matrices of pixels with a size of $0.52 \mu \mathrm{m}$ and 256 phase levels. At the experimental wavelength of the laser $(\lambda=632.8 \mathrm{~nm})$, the refractive index of the resist polymer was assessed to be $n_{\mathrm{R}}=1.679$ [as measured by spectroscopic ellipsometry (J.A. Woollam VASE, $0.3 \mathrm{~nm}$ spectral resolution, $0.005^{\circ}$ angular resolution)]. From Eq. (16), the maximal depth of the surface-relief pattern was found to be $928.5 \mathrm{~nm}$, with a thickness resolution $\Delta d=3.64 \mathrm{~nm}$. By using custom numerical codes, the phase patterns of the simulated optics were converted into a 3D multilevel structure, which was in turn transformed into a map of electronic doses. A dose correction for compensating for the proximity effects was required to both match the layout depth with the fabricated relief and obtain a good shape definition in correspondence to phase discontinuities. The resist exposure was performed with a JBX-6300FS JEOL EBL machine in high-resolution mode, $12 \mathrm{MHz}$, generating at $100 \mathrm{KeV}$ and $100 \mathrm{pA}$ an electron beam with a diameter of $2 \mathrm{~nm}$, providing a resolution down to $5 \mathrm{~nm}$. After exposure, a cross-linking baking was performed at $100^{\circ} \mathrm{C}$ for $60 \mathrm{~min}$, followed by a post-baking process at $70^{\circ} \mathrm{C}$ for 2 hours to improve the surface roughness. Finally, samples were developed for $510 \mathrm{~s}$ in AR 300-47 developer (Allresist). After development, the optical elements were gently rinsed in deionized water and blow-dried under nitrogen flux.

\section{Optical characterization}

Integer OAM beams were generated employing an LCoS SLM (PLUTO-NIR-010-A, Holoeye) using a phase and amplitude modulation technique ${ }^{39}$. The Gaussian beam $\left(\lambda=632.8 \mathrm{~nm}\right.$, beam waist $w_{0}=240 \mu \mathrm{m}$, and power of $0.8 \mathrm{~mW}$ ) emitted by a HeNe laser (HNR008R, Thorlabs) was linearly polarized and expanded with a telescope $\left(f_{1}=3.5 \mathrm{~cm}, f_{2}=12.5 \mathrm{~cm}\right)$ before illuminating the SLM display. A second telescope $\left(f_{3}=25.0 \mathrm{~cm}, f_{4}=12.5 \mathrm{~cm}\right)$ was used to isolate and image the first order encoded mode onto the optical element under test, mounted on a six-axis kinematic mount (K6XS, Thorlabs). A 50:50 beam splitter was used to split the beam and check the input beam profile with the first camera (DCC1545M,
Thorlabs). The beam illuminated the first zone of the sample, performing either $n$-fold multiplication or division. A mirror was placed on a kinematic mount (KM100, Thorlabs), and its position could be finely controlled with a micrometric translator (TADC-651, Optosigma). The distance from the multiplier/divider was equal to half the focal length of the first element, i.e., $1 \mathrm{~cm}$. After backreflection, the transformed beam illuminated the optics again on the phase-correcting zones, and the Fouriertransformed beam was collected by a second CMOS camera (DCC1545M, Thorlabs), placed at the back focal plane of a lens with $f=10.0 \mathrm{~cm}$. To check the OAM content of the output beams, a Mach-Zehnder interferometric bench was added to the described optical setup, as depicted in Fig. 6a.

\section{Acknowledgements \\ This work was supported by project Vortex 2 from CEPOLISPE.}

\section{Author details}

'Department of Physics and Astronomy 'G. Galilei', University of Padova, via Marzolo 8, 35131 Padova, Italy. ${ }^{2}$ LaNN, Laboratory for Nanofabrication of Nanodevices, EcamRicert, Corso StatiUniti 4, 35127 Padova, Italy. ${ }^{3}$ CNR-INFM TASC IOM National Laboratory, S.S. 14 Km 163.5, 34012 Trieste, Italy

\section{Authors' contributions}

G.R. conceived the idea, performed the design and optimization of the phase pattern, and conducted optical characterization. M.M. performed EBL fabrication and both optical microscopy and SEM. F.R. addressed the aims of the project, supervised the work and managed the laboratory. All authors discussed and contributed to the writing of the manuscript.

Conflict of interest

The authors declare that they have no conflict of interest.

Supplementary information is available for this paper at https://doi.org/ 10.1038/s41377-019-0222-2.

Received: 12 April 2019 Revised: 5 November 2019 Accepted: 11 November 2019

Published online: 05 December 2019

\footnotetext{
References

1. Allen, L. et al. Orbital angular momentum of light and the transformation of Laguerre-Gaussian laser modes. Phys. Rev. A 45, 8185-8189 (1992).

2. Padgett, M. J. Orbital angular momentum 25 years on. Opt. Express 25, 11265-11274 (2017).

3. Padgett, M. \& Bowman, R. Tweezers with a twist. Nat. Photonics 5, 343-348 (2011).

4. Ritsch-Marte, M. Orbital angular momentum light in microscopy. Philos. Trans. R. Soc. A: Math., Phys. Eng. Sci. 375, 20150437 (2017).

5. Vicidomini, G., Bianchini, P. \& Diaspro, A. STED super-resolved microscopy. Nat. Methods 15, 173-182 (2018).

6. Mari, E. et al. Sub-Rayleigh optical vortex coronagraphy. Opt. Express 20, 2445-2451 (2012).

7. Wang, J. Twisted optical communications using orbital angular momentum. Science China Physics, Mechanics \&. Astronomy 62, 34201 (2019).

8. Mirhosseini, M. et al. High-dimensional quantum cryptography with twisted light. N. J. Phys. 17, 033033 (2015).

9. Ruffato, G. et al. Design, fabrication and characterization of computer generated holograms for anti-counterfeiting applications using OAM beams as light decoders. Sci. Rep. 7, 18011 (2017).

10. Khonina, S. N. et al. The phase rotor filter. J. Mod. Opt. 39, 1147-1154 (1992).
} 
11. Massari, M. et al. Fabrication and characterization of high-quality spiral phase plates for optical applications. Appl. Opt. 54, 4077-4083 (2015).

12. Sacks, Z. S., Rozas, D. \& Swartzlander, G. A. Holographic formation of opticalvortex filaments. J. Optical Soc. Am. B 15, 2226-2234 (1998).

13. Lei, T. et al. Massive individual orbital angular momentum channels for multiplexing enabled by Dammann gratings. Light Sci. Appl. 4, e257 (2015).

14. Marrucci, L., Manzo, C. \& Paparo, P. Pancharatnam-Berry phase optical elements for wave front shaping in the visible domain: switchable helical mode generation. Appl. Phys. Lett. 88, 221102 (2006).

15. Fickler, R. et al. Interface between path and orbital angular momentum entanglement for high-dimensional photonic quantum information. Nat. Commun. 5, 4502 (2014).

16. Li, W. Z. et al. Rapidly tunable orbital angular momentum (OAM) system for higher order Bessel beams integrated in time (HOBBIT). Opt. Express 27, 3920-3934 (2019).

17. Chen, M. L. N., Jiang, L. J. \& Sha, W. E. I. Orbital angular momentum generation and detection by geometric-phase based metasurfaces. Appl. Sci. 8, 362 (2018).

18. Hell, S. W. \& Wichmann, J. Breaking the diffraction resolution limit by stimulated emission: stimulated-emission-depletion fluorescence microscopy. Opt. Lett. 19, 780-782 (1994).

19. Yu, S. Y. Potentials and challenges of using orbital angular momentum communications in optical interconnects. Opt. Express 23, 3075-3087 (2015).

20. Wang, J. et al. Terabit free-space data transmission employing orbital angular momentum multiplexing. Nat. Photonics 6, 488-496 (2012)

21. Bozinovic, N. et al. Terabit-scale orbital angular momentum mode division multiplexing in fibers. Science 340, 1545-1548 (2013).

22. Scaffardi, M. et al. Interconnection network architectures based on integrated orbital angular momentum emitters. Opt. Commun. 408, 63-67 (2018).

23. Ruffato, G. et al. Electrically activated spin-controlled orbital angular momentum multiplexer. Appl. Phys. Lett. 113, 011109 (2018).

24. Willner, A. E. et al. Orbital-angular-momentum-based reconfigurable optical switching and routing. Photonics Res. 4, B5-B8 (2016).

25. Liu, J. \& Wang, J. Demonstration of reconfigurable joint orbital angular momentum mode and space switching. Sci. Rep. 6, 37331 (2016).

26. García-Escartín, J. C. \& Chamorro-Posada, P. Quantum multiplexing with the orbital angular momentum of light. Phys. Rev. A 78, 062320 (2008).

27. García-Escartín, J. C. \& Chamorro-Posada, P. Universal quantum computation with the orbital angular momentum of a single photon. J. Opt. 13, 064022 (2011).

28. Potoček, V. et al. Quantum Hilbert hotel. Phys. Rev. Lett. 115, 160505 (2015).

29. Zhao, Z. et al. Invited article: division and multiplication of the state order for data-carrying orbital angular momentum beams. APL Photonics 1, 090802 (2016).
30. Zhou, H. L. et al. Orbital angular momentum divider of light. IEEE Photon. J. 9, 6500208 (2017)

31. Berkhout, G. C. G. et al. Efficient sorting of orbital angular momentum states of light. Phys. Rev. Lett. 105, 153601 (2010).

32. Lavery, M. P. J. et al. Refractive elements for the measurement of the orbital angular momentum of a single photon. Opt. Express 20, 2110-2115 (2012).

33. O'Sullivan, M. N. et al. Near-perfect sorting of orbital angular momentum and angular position states of light. Opt. Express 20, 24444-24449 (2012).

34. Ruffato, G. et al. Test of mode-division multiplexing and demultiplexing in free-space with diffractive transformation optics. Opt. Express 25, 7859-7868 (2017).

35. Ruffato, G. et al. A compact diffractive sorter for high-resolution demultiplexing of orbital angular momentum beams. Sci. Rep. 8, 10248 (2018).

36. Takashima, S., Kobayashi, H. \& Iwashita, K. Integer multiplier for orbita angular momentum of light using circular-sector transformation. Preprint at https:/arxiv.org/abs/1902.10472, https://journals.aps.org/pra/accepted/ 8d070Y3aOfa19863589627f0451c8001552155743\#abstract (2019).

37. Hossack, W. J., Darling, A. M. \& Dahdouh, A. Coordinate transformations with multiple computer-generated optical elements. J. Mod. Opt. 34, 1235-1250 (1987).

38. Ruffato, G., Massari, M. \& Romanato, F. Diffractive optics for combined spatialand mode- division demultiplexing of optical vortices: design, fabrication and optical characterization. Sci. Rep. 6, 24760 (2016).

39. Rosales-Guzmán, C. \& Forbes, A. How to shape light with spatial light modulators (SPIE Press, 2017).

40. Lavery, M. P. J. et al. Efficient measurement of an optical orbital-angularmomentum spectrum comprising more than 50 states. N. J. Phys. 15, 013024 (2013).

41. Berry, M. V. \& McDonald, K. T. Exact and geometrical optics energy trajectories in twisted beams. J. Opt. A 10, 035005 (2008).

42. Liu, C. M. et al. Discrimination of orbital angular momentum modes of the terahertz vortex beam using a diffractive mode transformer. Opt. Express 24, 12534-12541 (2016).

43. McMorran, B. J. et al. Origins and demonstrations of electrons with orbital angular momentum. Philos. Trans. R. Soc. 375, 20150434 (2017).

44. Harris, J. et al. Structured quantum waves. Nat. Phys. 11, 629-634 (2015).

45. Grillo, $V$. et al. Measuring the orbital angular momentum spectrum of an electron beam. Nat. Commun. 8, 15536 (2017).

46. McMorran, B. J., Harvey, T. R. \& Lavery, M. P. J. Efficient sorting of free electron orbital angular momentum. N. J. Phys. 19, 023053 (2017)

47. Li, J. C., Peng, Z. J. \& Fu, Y. C. Diffraction transfer function and its calculation of classic diffraction formula. Opt. Commun. 280, 243-248 (2007). 